УДК 517.982

N. M. BAZIV, O. B. Hrybel

\title{
ON THE ALGEBRAIC DIMENSION OF RIESZ SPACES
}

\begin{abstract}
N. M. Baziv, O. B. Hrybel. On the algebraic dimension of Riesz spaces, Mat. Stud. 56 (2021), $67-71$.

We prove that the algebraic dimension of an infinite dimensional $C$ - $\sigma$-complete Riesz space (in particular, of a Dedekind $\sigma$-complete and a laterally $\sigma$-complete Riesz space) with the principal projection property which either has a weak order unit or is not purely atomic, is at least continuum. A similar (incomparable to ours) result for complete metric linear spaces is well known.
\end{abstract}

For necessary information on Riesz spaces, we refer the reader to [1]. A linear basis of a vector space having some additional structure (like norm, metric, topology, order) is called a Hamel basis in order to distinguish this type of bases from the other ones (Schauder bases, order bases, ect.). More precisely, a system of elements $\left(x_{i}\right)_{i \in I}$ of a vector space $X$ is called a Hamel basis (or linear basis), if for every $x \in X$ there exists a unique system of finitely nonzero reals $\left(a_{i}\right)_{i \in I}$ such that $x=\sum_{i \in I} a_{i} x_{i}$. As an exercise, one can prove that the Hamel bases are exactly the maximal linearly independent systems in $X$. An application of Zorn's lemma yields that every linearly independent system can be extended to a Hamel basis. A nice introduction to Hamel bases can be found in [6]. Every two Hamel bases of a vector space $X$ have the same cardinality [6, p. 3], which is called the algebraic dimension of $X$.

The present note is devoted to complete a chain of the results by different authors asserting that, the algebraic dimension of an infinite dimensional complete (in some sense) vector space with an additional structure cannot be strictly less than continuum. A short proof that any Hamel basis of an infinite dimensional Banach space has at least continuum many elements was provided by H. E. Lacey in [7]. A more general result by T. O. Banakh and A. M. Plichko [2] asserts the same for the setting of complete metric linear spaces (as was mentioned by the authors later in [3], one can deduce their result from [4, Proposition VIII.2.2] by C. Bessaga and A. Pełczyński).

In order to achieve maximal generality in the theorem statement, we need some information. Given a Riesz space $E$, we consider the lateral order on $E$ by setting $x \sqsubseteq y$ for any $x, y \in E$ if and only if $x$ is a fragment of $y$, that is, $x \perp(y-x)$. It is a partial order on $E$ (see [9] for more details about this order), which coincides with the usual order $\leq$ on the positive cone $E^{+}$. A subset $A \subseteq E$ is said to be laterally bounded if there is $e \in E$ such that $x \sqsubseteq e$ for all $x \in A$ (the lateral boundedness is considered from above only because every subset is laterally bounded from below by zero). The lateral supremum of a subset $A \subseteq E$ is

2010 Mathematics Subject Classification: 46A16, 46A40, 54F05.

Keywords: Riesz space; algebraic dimension; lateral order.

doi:10.30970/ms.56.1.67-71

(C) N. M. Baziv, O. B. Hrybel, 2021 
defined to be the supremum (if exists) of $A$ with respect to the lateral order, and denoted by $\bigcup A$.

A Riesz space $E$ is said to be laterally complete if every disjoint family from $E^{+}$has a supremum. Equivalently, $E$ is laterally complete if and only if every disjoint family from $E$ has a lateral supremum [9, Proposition 5.3]. A Riesz space $E$ is said to be $C$-complete if every nonempty laterally bounded subset $G$ of $E$ has a lateral supremum $\bigcup G \in E$. The notion of $C$-completeness is the most general one: if a Riesz space $E$ is either Dedekind complete or laterally complete then $E$ is $C$-complete [9, Corollary 5.8]. On the other hand, the Banach lattice $C[0,1]$ is a $C$-complete Riesz space which is neither Dedekind complete, nor laterally complete.

We consider the $\sigma$-versions of the above notions, which are much more general. A Riesz space $E$ is said to be

- laterally $\sigma$-complete if every disjoint sequence from $E^{+}$has a supremum;

- $C$ - $\sigma$-complete if every nonempty laterally bounded at most countable subset $G$ of $E$ has a lateral supremum $\bigcup G \in E$.

Using the same proof as in [9, Corollary 5.8] with some little adjustments, one can show that if a Riesz space $E$ is either Dedekind $\sigma$-complete or laterally $\sigma$-complete then $E$ is $C$ - $\sigma$-complete.

Theorem 1. Let $E$ be a $C$ - $\sigma$-complete Riesz space admitting a disjoint laterally bounded sequence of nonzero elements. Then the algebraic dimension of $E$ is at least continuum.

We say that subsets $A, B$ of $\mathbb{N}$ are almost disjoint provided $A \cap B$ is finite. One can show that there exists a family $\left(A_{x}\right)_{x \in \mathbb{R}}$ of pairwise almost disjoint infinite subsets of $\mathbb{N}$, which we use in our proof below (see e.g. [5]).

Proof. Let $\left(e_{n}\right)_{n=1}^{\infty}$ be a laterally bounded sequence of nonzero elements. With no loss of generality we may and do assume that $e_{n}>0$ for all $n$, otherwise consider $\left|e_{n}\right|$ instead of $e_{n}$. Define a family $\left(f_{x}\right)_{x \in \mathbb{R}}$ in $E$ as follows. Given any $x \in \mathbb{R}$, we set

$$
f_{x}=\sup _{n \in A_{x}} e_{n}, x \in \mathbb{R}
$$

( $f_{x}$ is well defined by the theorem assumptions, because the lateral supremum coincides with the usual supremum on $\left.E^{+}\right)$. It is enough to prove that the family $\left(f_{x}\right)_{x \in \mathbb{R}}$ is linearly independent. Assuming the contrary, we obtain a linearly dependent finite subsystem $\left(f_{x_{k}}\right)_{k=1}^{m}, m \in \mathbb{N}$. Let $\left(\lambda_{k}\right)_{k=1}^{m}$ be real numbers not all equal zero such that $\sum_{k=1}^{m} \lambda_{k} f_{x_{k}}=0$. Say, $\lambda_{m} \neq 0$. Then

$$
f_{x_{m}}=-\lambda_{m}^{-1} \sum_{k=1}^{m-1} \lambda_{k} f_{x_{k}}
$$

Set

$$
A:=A_{x_{m}} \backslash\left(\bigcup_{k=1}^{m-1} A_{x_{k}}\right)=A_{x_{m}} \backslash\left(\bigcup_{k=1}^{m-1}\left(A_{x_{m}} \cap A_{x_{k}}\right)\right)
$$

Since $A_{x_{m}}$ is infinite and $A_{x_{m}} \cap A_{x_{k}}$ is finite for all $k \in\{1, \ldots, m-1\}$, we obtain that $A \neq \varnothing$. Fix some $j \in A$. Observe that $j \in A_{x_{m}}$ implies $e_{j} \wedge f_{x_{m}}=e_{j}$, and $j \notin A_{x_{k}}$ implies $e_{j} \wedge f_{x_{k}}=0$ for $k \in\{1, \ldots, m-1\}$. Since the disjoint complement to any subset of a Riesz space is a 
band (in particular, is a linear subspace) [1, p. 34], one has that $\left\{e_{j}\right\}^{d}$ is a linear subspace and hence $e_{j} \wedge\left(-\lambda_{m}^{-1} \sum_{k=1}^{m-1} \lambda_{k} f_{x_{k}}\right)=0$. Therefore, taking infimum of $e_{j}$ with both sides of equality (2), we obtain

$$
0<e_{j}=e_{j} \wedge f_{x_{m}}=e_{j} \wedge\left(-\lambda_{m}^{-1} \sum_{k=1}^{m-1} \lambda_{k} f_{x_{k}}\right)=0,
$$

a contradiction which proves the theorem.

Corollary 1. Let $E$ be a Dedekind $\sigma$-complete Riesz space admitting a disjoint order bounded sequence of nonzero elements. Then the algebraic dimension of $E$ is at least continuum.

Although the same proof as that of Theorem 1 with minor adjustments will do for Corollary 1, one can formally obtain Corollary 1 as a consequence of Theorem 1 by showing that an order bounded sequence in a Dedekind $\sigma$-complete Riesz space is laterally bounded.

Corollary 2. Let $E$ be a laterally $\sigma$-complete Riesz space admitting a disjoint sequence of nonzero elements. Then the algebraic dimension of $E$ is at least continuum.

Next we provide a simple example showing that the lateral boundedness assumption on a sequence in Theorem 1 is essential, as well as the order bounded assumption in Corollary 1. Let $c_{00}$ denote the Riesz space of all eventually zero real sequences $x=\left(\xi_{1}, \xi_{2}, \ldots\right)$ (that is, $\left.\left(\exists n_{0} \in \mathbb{N}\right)\left(\forall n \geq n_{0}\right) \xi_{n}=0\right)$ with the coordinate-wise order.

Example 1. The Riesz space $c_{00}$ is Dedekind complete and the unit vector basis $\left(e_{n}\right)_{n=1}^{\infty}$ forms a countable Hamel basis of $c_{00}$ being a disjoint sequence of nonzero elements.

The following one-dimensional modification of $c_{00}$ shows that the $C$ - $\sigma$-completeness assumption in Theorem 1 is essential, as well as the Dedekind $\sigma$-completeness assumption in Corollary 1. Another pathological conclusion of the same example is that, a Riesz space which is a direct sum of two Dedekind complete Riesz subspaces need not be Dedekind complete.

Example 2. Let $E$ denote the Riesz space of all eventually constant sequences of real numbers with the coordinate-wise order. Then $E$ possesses the following properties.

1. $E$ equals the direct sum of its Dedekind complete Riesz subspaces $c_{00}$ and $c_{\mathbb{R}}:=$ $\{(\lambda, \lambda, \ldots): \lambda \in \mathbb{R}\}$, that is, $E=c_{00} \oplus c_{\mathbb{R}}$.

2. $E$ has the principal projection property and is not Dedekind $\sigma$-complete. Moreover, $E$ is not $C$ - $\sigma$-complete.

3. The unit vectors $\left(e_{n}\right)_{n=1}^{\infty}$ form a laterally bounded (and hence, order bounded) disjoint sequence of nonzero elements in $E$.

4. The sequence $\left(e_{n}\right)_{n=0}^{\infty}$, where $e_{0}=(1,1, \ldots) \in c_{\mathbb{R}}$, is a countable Hamel basis of $E$.

Proof. We prove item 2 only, because the rest ones are obvious. First we show that $E$ has the principal projection property. Fix any $x=\left(a_{1}, a_{2}, \ldots\right) \in E$. Set $J:=\left\{j \in \mathbb{N}: a_{j}=0\right\}$ and $I:=\mathbb{N} \backslash J$. Then for the principal band $B_{x}$ generated by $x$ one has

$$
B_{x}=\left\{\left(\xi_{1}, \xi_{2}, \ldots\right) \in E:(\forall j \in J) \xi_{j}=0\right\}, B_{x}^{d}=\left\{\left(\xi_{1}, \xi_{2}, \ldots\right) \in E:(\forall i \in I) \xi_{i}=0\right\}
$$


and $B_{x} \oplus B_{x}^{d}=E$. So, $E$ has the principal projection property.

Now we prove that $E$ is not $C$ - $\sigma$-complete. The sequence $\left(e_{2 n}\right)_{n=1}^{\infty}$ is laterally bounded, for instance, by $e_{0}$. Show that this sequence has no lateral supremum. Indeed, let $z$ be any lateral upper bound of $\left(e_{2 n}\right)_{n=1}^{\infty}$. Decompose $z=x+y$, where $x \in c_{00}$ and $y \in c_{\mathbb{R}}$, say, $x=\left(a_{1}, \ldots, a_{i}, 0,0, \ldots\right)$, where $i \in \mathbb{N}$ and $y=\lambda e_{0}$, where $\lambda \in \mathbb{R}$. Then $z^{\prime}:=z-e_{2 i+1}$ is another lateral upper bound of $\left(e_{2 n}\right)_{n=1}^{\infty}$ which is strictly less than $z$. This confirms that $E$ is not $C$ - $\sigma$-complete.

Now we provide necessary information on atoms. An element $u \neq 0$ of a Riesz space $E$ is called

- an atom, whenever $0 \leq x \leq|u|, 0 \leq y \leq|u|$ and $x \wedge y=0$ imply that either $x=0$ or $y=0$

- a weak atom, if $u$ has no proper fragment, that is, for every $x \sqsubseteq u$ either $x=0$ or $x=u$.

On can easily show that if $u \in E$ is an atom then either $u>0$ or $u<0$ [8, Lemma 26.2 (i)]. On the other hand, if $u$ is an atom then $-u$ is obviously an atom as well. So, for many purposes, it is enough to consider positive atoms only. Every atom $0<u \in E$ is a weak atom, and if $E$ has the principal projection property then every weak atom in $E$ is an atom [10, Proposition 1.3].

A Riesz space is said to be atomless if it has no atom. We say that a Riesz space $E$ is purely atomic if there is a collection $\left(u_{i}\right)_{i \in I}$ of atoms in $E^{+}$, called a generating collection of atoms, such that $u_{i} \perp u_{j}$ for $i \neq j$ and for every $x \in E$ if $|x| \wedge u_{i}=0$ for each $i \in I$ then $x=0$.

By [10, Proposition 1.6], any Riesz space $E$ with the principal projection property has a decomposition to mutually complemented bands $E=E_{0} \oplus E_{1}$ where $E_{0}$ is a purely atomic vector lattice and $E_{1}$ is an atomless vector lattice.

By $P_{x}$ we denote the order projection of $E$ onto the principal band generated by a projection element $x$ of $E$. By [1, Prop.1.47], $P_{x} y=\sup _{m \in \mathbb{N}}(y \wedge m|x|)$ for all $y \in E^{+}$. It is well known and easily seen that $P_{x} y \sqsubseteq y$ for all $y \in E$.

Theorem 2. Let $E$ be an infinite dimensional $C$ - $\sigma$-complete Riesz space with the principal projection property. If $E$ either has a weak order unit or is not purely atomic then every Hamel basis of $E$ has at least continuum many elements.

Proof. By Theorem 1, it is enough to prove the existence of a laterally bounded disjoint sequence of nonzero elements. Consider cases.

1. Assume $E$ has a weak order unit $e>0$ and $E$ is purely atomic with a generating collection of positive atoms $\left(u_{i}\right)_{i \in I}$. Since $E$ is infinite dimensional, the index set $I$ is infinite. With no loss of generality, we assume that $\mathbb{N} \subseteq I$. Since $e$ is a weak order unit, we have that $e \wedge u_{n}>0$ and hence,

$$
e_{n}:=P_{u_{n}} e=\sup _{m \in \mathbb{N}}\left(e \wedge m u_{n}\right)>0
$$

for all $n \in \mathbb{N}$.

Now we show that for every $n \in \mathbb{N}$ there exists $\lambda_{n}>0$ such that $e_{n}=\lambda_{n} u_{n}$. Fix any $n, m \in \mathbb{N}$. A direct verification shows that $e \wedge m u_{n}$ is an atom. By [8, Theorem 26,4 (ii)], any two atoms in a Riesz space are either disjoint or linearly dependent. So, since $e \wedge m u_{n}$ 
is not disjoint to $u_{n}$, there is $\alpha_{m, n}>0$ such that $e \wedge m u_{n}=\alpha_{m, n} u_{n}$. Then for any $n \in \mathbb{N}$ the number $\lambda_{n}=\sup _{m \in \mathbb{N}} \alpha_{m, n}$ is finite, because

$$
e_{n}=\sup _{m \in \mathbb{N}}\left(e \wedge m u_{n}\right)=\sup _{m \in \mathbb{N}} \alpha_{m, n} u_{n}=\lambda_{n} u_{n}
$$

Consequently, $\left(e_{n}\right)_{n=1}^{\infty}$ is a disjoint sequence of atoms. Since $P_{u_{n}} e \sqsubseteq e$ for all $n \in \mathbb{N}$, this sequence is laterally bounded by $e$. By (3) of Theorem 1, every Hamel basis of $E$ has at least continuum many elements.

2. Assume $E$ is not purely atomic. Let $E_{1}$ be a nontrivial atomless band of $E$ and $0<e \in E_{1}$. Decompose $e$ into disjoint nonzero fragments $e=e_{1}+e_{1}^{\prime}$. Then decompose $e_{1}^{\prime}$ into nonzero disjoint fragments $e_{1}^{\prime}=e_{2}+e_{2}^{\prime}$. Arguing like that, we construct a disjoint sequence of nonzero fragments of $e$. By (3) of Theorem 1, every Hamel basis of $E$ has at least continuum many elements.

Acknowledgments. The authors are thankful to Professor M. M. Popov for his advices and valuable discussions on the subject, and to the anonymous referee for paying our attention to works [3] and [4].

\section{REFERENCES}

1. C.D. Aliprantis, O. Burkinshaw, Positive operators, Springer, Dordrecht, 2006.

2. T. Banakh, A. Plichko, The algebraic dimension of linear metric spaces and Baire properties of their hyperspaces, RACSAM. Rev. R. Acad. Cienc. Exactas Fis. Nat. Ser. A Mat., 100 (2006), №1-2, 31-37.

3. T. Banakh, A. Plichko, Letter to editors from T. Banakh, A. Plichko, RACSAM. Rev. R. Acad. Cienc. Exactas Fis. Nat. Ser. A Mat., 102 (2008), №2, 203.

4. C. Bessaga, A. Pełczyński, Selected topics in infinite-dimensional topology, Monografie Matematyczne, Warszawa, 1975.

5. J.R. Buddenhagen, Classroom notes: subsets of a countable set, Amer. Math. Monthly 78 (1971), №5, $536-537$.

6. M.M. Day, Normed linear spaces, Springer-Verlag, Berlin-Heidelberg-New York, 1973.

7. H.E. Lacey, The Hamel dimension of any infinite dimensional separable Banach space is c,, Amer. Math. Monthly, 80 (1973), №3, 298.

8. W.A.J. Luxemburg, A.C. Zaanen, Riesz spaces. Vol. 1, North Holland Publ. Comp., Amsterdam-London, 1971.

9. V. Mykhaylyuk, M. Pliev, M. Popov, The lateral order on Riesz spaces and orthogonally additive operators, Positivity 25 (2021), №2, 291-327. DOI: 10.1007/s11117-020-00761-x.

10. V. Mykhaylyuk, M. Pliev, M. Popov, O. Sobchuk, Dividing measures and narrow operators, Studia Math., 231 (2015), №2, 97-116.

Vasyl Stefanyk Precarpathian National University

Ivano-Frankivsk, Ukraine

nataliia.baziv@pnu.edu.ua

olha.hrybel@pnu.edu.ua 